\title{
Psychological adjustment in children with episodic migraine: a population-based study
}

\author{
Marco A. Arruda ${ }^{1}$ and Renato Arruda ${ }^{2}$ \\ 1. Instituto Glia, Ribeirão Preto, SP, Brazil \\ 2. Universidade Estadual de Campinas, Campinas, SP, Brazil
}

\begin{abstract}
We investigated psychological adjustment in a preadolescent pediatric population as a function of headache diagnosis. Children from a city public education system were enrolled in this study. Parents were interviewed using validated headache questionnaires and the Strengths and Difficulties Questionnaire (SDQ), which measures psychological adjustment. Crude and adjusted prevalence ratios were obtained using a binary regression model. The relative risk [RR] of SDQ items and scores were modeled as a function of headache diagnosis in adjusted analyses. Multivariate models estimated determinants of psychological adjustment characteristics in children with migraine. The sample consisted of 846 children ( $65.9 \%$ of the target sample) from 5 to 12 years old $(50.5 \%$ girls). Relative to children without headache, children with episodic migraine (EM) were more likely to have abnormal scores on the following SDQ scales: emotional symptoms $(\mathrm{RR}=3.43,95 \%$ confidence interval $[\mathrm{CI}]=2.51-4.69)$, conduct problems $(\mathrm{RR}=1.96,95 \% \mathrm{CI}=1.37-2.79)$, total difficulties $(\mathrm{RR}=2.23,95 \% \mathrm{CI}=1.59-3.13)$, and total impact $(\mathrm{RR}=$ $2.85,95 \% \mathrm{CI}=1.15-7.11)$. The multivariate analysis showed that total difficulties in psychological adjustment in children with EM were significantly influenced by headache frequency $(p<.05)$, analgesic intake $(p<.001)$, and the occurrence of nausea $(p$ $<.01)$ and vomiting $(p<.05)$ in headache attacks. To the best of our knowledge, this is the first study reported in the literature to identify determinants of the association between migraine and difficulties in psychological adjustment in preadolescent children. Providers and educators should be aware of this association, and studies that address causality should be conducted. Keywords: headache, migraine, psychological adjustment, SDQ, children, epidemiology.
\end{abstract}

Received 27 March 2012; received in revised form 27 July 2012; accepted 25 September 2012. Available online 07 March 2014.

\section{Introduction}

Headache is one of the most common pain and neurological symptoms in childhood (Goodman \& McGrath, 1991; Perquin et al., 2001). The lifetime prevalence of headache in children reaches a striking rate of $82 \%$ (Arruda et al., 2010a). Migraine and tension-type headache, the prototypical primary headaches, are common in the preadolescent pediatric population (Abu-Arafeh, Razak, Sivaraman, \& Graham, 2010). When the International Headache Society criteria are strictly followed, the prevalence of migraine in children ranges from $3.3 \%$ to $21.4 \%$, increasing from early childhood to adolescence (AbuArafeh et al., 2010). The burden of pediatric headaches is best characterized for migraine, which impacts the child's quality of life (Powers, Patton, Hommel, \&

Marco A. Arruda, M.D., Ph.D., Instituto Glia, Ribeirão Preto, SP, Brazil. Renato Arruda, Faculdade de Ciências Médicas, Universidade Estadual de Campinas, SP, Brazil. Correspondence regarding this article should be directed to: Marco A. Arruda, Av. Braz Olaia Acosta, 727, s. 310, Ribeirão Preto, SP, CEP 14026-040, Brazil. Phone: +55 (16) 39119234. Fax: +55 (16) 39119234. E-mail: arruda@institutoglia.com.br
Hershey, 2003), school attendance (Abu-Arefeh, \& Russell, 1994; Arruda, \& Bigal, 2012a), and school performance (Arruda \& Bigal, 2012a). Pediatric headache can also disrupt the family (Galli, Canzano, Scalisi, \& Guidetti, 2009). Research suggests that the impact of migraine is influenced by several factors including severity of pain and its associated symptoms, frequency of pain, anticipatory anxiety, adequacy of treatment, and comorbidities (Arruda \& Bigal, 2012a; Bigal, Krymchantowski, \& Lipton, 2009; Lipton $\&$ Silberstein, 1994). Nonetheless, the influence of established comorbidities of psychological symptoms on the burden of primary headaches is still poorly studied in children and adolescents.

Clinical (Bruijn, Locher, Passchier, Dijkstra, \& Arts, 2010; Galli et al., 2007; Guidetti et al., 1998; Vannatta, Getzoff, Powers, Noll, Gerhardt, \& Hershey, 2008) and populational (Anttila, Sourander, Metsahonkala, Aromaa, Helenius, \& Sillanpaa, 2004; Arruda \& Bigal, 2012b; Virtanen et al., 2004) studies suggest that children with migraine are more likely to have somatic, anxiety, and depressive symptoms relative to children without headaches. Limited findings also suggest that pediatric migraine is associated with impaired attention span (Virtanen et al., 2004) and hyperactivity-impulsivity but 
not with fully developed attention-deficit/hyperactivity disorder (ADHD, Arruda, Guidetti, Galli, Albuquerque, \& Bigal, 2010b). Representative data for other primary headaches are scarce.

In his seminal work with children who suffered from migraine, Bille (1962) found that children with migraine were "more sensitive," "more tidy," "less physically enduring," and "more vulnerable to frustration" than controls (Bille, 1962). The concept of a "migrainous personality" was later proposed by Saper (1983), describing a migraine patient as having the following characteristics: "compulsive, perfectionistic, rigid, and achievement driven elements, often accompanied by internalized anger and excessive self-control." However, the association between these characteristics and migraine has not yet been confirmed by scientific evidence. Findings from more recent studies suggest a possible association between migraine and other psychological characteristics in children and adolescents such as shyness, emotional rigidity, tendency to repress anger and aggression, and a poor level of adjustment (Andrasik, Kabela, Quinn, Attanasio, Blanchard, \& Rosenblum, 1988; Lanzi et al., 2001; Mazzone, Vitiello, Incorpora, \& Mazzone, 2006).

Adjustment in psychology can be defined as a process of the progressive modification of thoughts and behavior to balance conflicting needs or needs against obstacles in the environment. Although it largely reflects the difficulties that individuals have in mastering or adapting to adverse conditions, personality characteristics are important factors that influence individual responses to life events (Sarbin, 1940). Adjustment disorder occurs when there is an inability to make normal adjustments to needs or stress in the environment (American Psychiatric Association, 1994).

Over the last decades, some studies have suggested that children with chronic physical disorders exhibit a higher level of difficulty in psychological adjustment and are more prone to develop psychopathology (Lavigne \& Faier-Routman, 1992). Therefore, a greater understanding of psychological functioning in children with migraine is of interest. To address this need, we have taken advantage of a large epidemiological study (Attention-Brazil Project) in which children were identified at schools, and parents were interviewed to investigate psychological adjustment assessed by the Strengths and Difficulties Questionnaire (SDQ) as a function of headache status and diagnosis.

\section{Methods}

\section{Overview}

This study is part of a larger project designed to establish inception cohorts for studying disorders that may impact learning in preadolescent children. It was built from a virtual network of professionals that began in 2006 with a nonprofit academic organization called "Aprender Criança" (Learning the Child; Arruda, 2009). Currently, a total of 4,500 members are registered in the organization. Of these members, $81 \%$ are teachers from the educational system in Brazil.

To achieve this aim, a pilot study was initially conducted in which all of the children registered in the school system of a city were selected. Their mothers and teachers were interviewed by one of the researchers of the present study. The details of this phase have been described elsewhere (Arruda, Guidetti, Galli, Albuquerque, \& Bigal, 2010a, b). The pilot study defined the validated questionnaires that would be used in the nationwide phase.

\section{Flow of the study}

In May 2009, we invited 124 teachers selected from 1,152 members of the organization who were registered to participate in the project. They were selected to representatively cover the Brazilian territory including rural and urban areas (Instituto Brasileiro de Geografia e Estatística, 2009). They all completed 4-h online training administered by one of the authors of the present study (MAA). During training, they were instructed on how to apply the study questionnaires (see below). We emphasize that using teachers to obtain health information is a well-accepted method for assessing mental health in preadolescent children (Akinbami, Liu, Pastor, \& Reuben, 2011; Epstein, Langberg, Lichtenstein, Kolb, Altaye, \& Simon, 2011). The teachers were not selected based on special interest in headache disorders, although they certainly had an interest in mental health because they voluntarily joined the community.

We present findings from the survey conducted in the city of Araquari in Santa Catarina state where four teachers were trained and conducted the interviews. The students were randomly identified and their parents were contacted. Those who agreed to participate were enrolled in the study. The interviews were performed at the end of October, which is close to the end of the school year in Brazil, which begins in February and ends in November.

\section{Geographic characterization and target sample}

According to the demographic census, the studied region covers an area with 24,814 inhabitants (year 2010). Of these, 23,151 (93.3\%) live in an urban area. Life expectancy is 72 years, and the Human Development Index is .767 , rates that are similar to the Brazilian rates (Instituto Brasileiro de Geografia e Estatística, 2009). Of the 3,214 children from 4 to 18 years of age registered in the public school system, $1,283(39.9 \%)$ were identified as our target sample and were invited to participate. Consent was obtained from 939 children (73.2\%), and complete demographic and headache information was obtained from 846 children $(65.9 \%)$. The participants' ages ranged from 5 to 12 years old (50.5\% girls). 


\section{Assessments}

Parents were interviewed by the teachers using a standardized questionnaire with 102 questions that assessed the following: (1) sociodemographic features, (2) past medical history of the child, (3) headaches, (4) parental perspective on school performance (not reported here), and (5) psychological adjustment.

The headache module of the questionnaire consisted of 14 questions that assessed the distinguishing features required for headache diagnosis in the children according to the International Classification of Headache Disorders, 2nd edition (ICHD-2; Headache Classification Subcommittee of the International Headache Society, 2004). The questionnaire was the validated Portuguese version used in American migraine studies (Lipton, Diamond, Reed, Diamond, \& Stewart, 2001) and has been extensively used in pediatric and adult populational studies in Brazil (Queiroz et al., 2008). Based on the response to the questionnaire, headache diagnoses were assigned by strictly following the ICHD-2 criteria. Children with EM fulfilled all of the required criteria for diagnosing EM. We did not differentiate migraine with and without aura. Probable migraine and chronic migraine were not considered in the analyses. Episodic tension-type headache (ETTH) encompassed children with frequent and infrequent ETTHs. Children in the "no headache" group did not meet the criteria for any primary headaches (including tension-type headaches).

Psychological adjustment was evaluated using the validated Brazilian version of the SDQ (Fleitlich-Bilyk \& Goodman, 2004; Goodman et al., 2005). The SDQ is a 25 -item instrument developed to assess emotional and behavioral problems from the view of the self (in adolescents or adults) or from the view of parents or teachers (Dickey \& Blumberg, 2004; Goodman, Lamping, \& Ploubidis, 2010). Validation studies and factor analyses have revealed that the SDQ can identify hyperactivity, inattention, emotional symptoms, peer problems, conduct problems, and prosocial behavior (Dickey \& Blumberg, 2004).

\section{Analyses}

Race and income were defined according to the definitions of the Brazilian Institute of Geography and Statistics (Instituto Brasileiro de Geografia e Estatística, 2009), which are adopted by the National Census. Race was dichotomized as white vs. non-white. The five income classes were defined based on total family income and do not reflect quintiles. They are determined based on the buying power for a basket of products and services that is defined by the institute and that correlates with socioeconomic characteristics. Classes A and B represent the upper classes. Classes $\mathrm{D}$ and $\mathrm{E}$ describe different levels of poverty. Class $\mathrm{C}$ is the middle class.

Sex-specific 1-year prevalence estimates of migraine and tension-type headache were derived by age, race, and income. To characterize the sample, descriptive statistics were performed. To estimate the relationship between sociodemographic characteristics and migraine status, cross tabulations were performed. Crude and adjusted prevalence ratios were obtained using a binary regression model. Prevalence ratios and 95\% confidence intervals (CIs) were used to compare specific categories (e.g., age categories or race) with the reference category.

\section{Multivariate analyses}

We initially developed nested multivariate models by considering the presence of migraine and an abnormal score on each of the six SDQ scales (emotional symptoms, conduct problems, hyperactivity, peer problems, total difficulties, and total impact) as dependent variables as a function of demographics (Model 1) and headache characteristics (mean duration, frequency and severity of the attacks, photophobia, phonophobia, nausea, vomiting, and analgesic abuse; Model 2). The level of significance adopted was 5\%. Statistical analysis was performed using SPSS 15.0 for Windows (SPSS Inc., Chicago, IL, USA).

\section{Investigation review board approval}

This study and the surveys received full approval from a university-based Ethics Review Committee (São José do Rio Preto Medical School, São Paulo, Brazil). Written informed consent was obtained from all parents.

\section{Results}

\section{Overview}

Table 1 displays the demographics of the participating sample and those without complete data. The overall participation rate was $-73 \%$, and complete data were obtained from $66 \%$ of the target sample and $90 \%$ of those who consented. Approximately $50 \%$ of the respondents were girls, and most were from the middle class (Class C, 56.1\%). The participation rates were very high for all categories, although they decreased as a function of decreased family income.

\section{Prevalence of headaches}

Among the participating children, 19.7\% did not report headaches (Table 2). Episodic migraine was diagnosed in $10.9 \%$ of the children $(11.5 \%$ of the girls and $10.3 \%$ of the boys). The prevalence of EM was not significantly associated with gender, age, race, or income class.

ETTH was diagnosed in $13 \%$ of the children $(11.5 \%$ of the girls and $14.6 \%$ of the boys). Although a higher prevalence was found in children aged 5 to 8 years (relative to those aged 9 to 12 years) and in boys, and a lower prevalence was found in children who came from lower income classes (relative to those from Classes $\mathrm{A}, \mathrm{B}$, and $\mathrm{C}$ ), the differences were not statistically significant.

The prevalence of headache increased as a function of age. Compared with children aged 5 to 8 years, the prevalence of "no headache" was significantly lower in 
Table 1. Target sample, consent obtained, and the final sample according to demographic features

\begin{tabular}{|c|c|c|c|c|c|c|}
\hline \multirow[b]{3}{*}{ Age group } & \multirow{3}{*}{$\begin{array}{l}\text { Target sample } \\
n\end{array}$} & & & \multicolumn{3}{|c|}{ Participation rate (\%) } \\
\hline & & \multicolumn{2}{|c|}{ Consent obtained } & \multirow{2}{*}{$\begin{array}{l}\text { Completed interviews } \\
n\end{array}$} & \multirow{2}{*}{$\begin{array}{l}\begin{array}{l}\text { Relative to } \\
\text { target sample }\end{array} \\
\%\end{array}$} & \multirow{2}{*}{$\begin{array}{l}\begin{array}{l}\text { Relative to those } \\
\text { who consented }\end{array} \\
\%\end{array}$} \\
\hline & & $n$ & $\%$ & & & \\
\hline $5-8$ years & 4,801 & 3,657 & 76.2 & 3,111 & 64.8 & 85.1 \\
\hline $9-12$ years & 3,798 & 2,788 & 73.4 & 2,560 & 67.4 & 91.8 \\
\hline \multicolumn{7}{|l|}{ Gender } \\
\hline Female & 4,259 & 3,186 & 74.8 & 2,794 & 65.6 & 87.7 \\
\hline Male & 4,340 & 3,259 & 75.1 & 2,877 & 66.3 & 88.3 \\
\hline \multicolumn{7}{|l|}{ School year } \\
\hline $1^{\text {st }}$ & 1,538 & 1,160 & 75.4 & 1,044 & 67.9 & 90.0 \\
\hline $2^{\text {nd }}$ & 2,384 & 1,884 & 79.0 & 1,674 & 70.2 & 88.9 \\
\hline $3^{\text {rd }}$ & 1,716 & 1,252 & 73.0 & 1,105 & 64.4 & 88.3 \\
\hline $4^{\text {th }}$ & 2,053 & 1,490 & 72.6 & 1,272 & 62.0 & 85.4 \\
\hline $5^{\text {th }}$ & 908 & 659 & 72.6 & 576 & 63.4 & 87.4 \\
\hline \multicolumn{7}{|l|}{ Race } \\
\hline White & 5,856 & 4,198 & 71.7 & 3,769 & 64.4 & 89.8 \\
\hline Non-white & 2,743 & 1,964 & 71.6 & 1,672 & 61.0 & 85.1 \\
\hline Non-respondents & & 283 & & 230 & & 81.3 \\
\hline \multicolumn{7}{|l|}{ Income class } \\
\hline A, B & 3,034 & 2,332 & 76.9 & 2,069 & 68.2 & 88.7 \\
\hline $\mathrm{C}$ & 4,347 & 3,234 & 74.4 & 2,856 & 65.7 & 88.3 \\
\hline $\mathrm{D}, \mathrm{E}$ & 1,218 & 879 & 72.2 & 746 & 61.2 & 84.9 \\
\hline \multicolumn{7}{|l|}{ Region } \\
\hline North & 278 & 172 & 61.9 & 134 & 48.2 & 77.9 \\
\hline Northeast & 1,245 & 912 & 73.3 & 712 & 57.2 & 78.1 \\
\hline Midwest & 405 & 298 & 73.6 & 188 & 46.4 & 63.1 \\
\hline Southeast & 3,778 & 2,837 & 75.1 & 2,542 & 67.3 & 89.6 \\
\hline South & 2,893 & 2,226 & 76.9 & 2,095 & 72.4 & 94.1 \\
\hline \multicolumn{7}{|c|}{ Population density } \\
\hline$<100,000$ & 3,245 & 2,567 & 79.1 & 2,220 & 68.4 & 86.5 \\
\hline $100,000-500,000$ & 3,546 & 2,589 & 73.0 & 2,365 & 66.7 & 91.3 \\
\hline$>500,000$ & 1,808 & 1,289 & 71.3 & 1,086 & 60.1 & 84.3 \\
\hline Total & 8,599 & 6,445 & 75.0 & 5,671 & 65.9 & 88.0 \\
\hline
\end{tabular}

children aged 9 to 12 years $(16.4 \%$ vs. $22.4 \%$, relative risk $[\mathrm{RR}]=.73,95 \% \mathrm{CI}=.55-.98)$.

\section{Psychological adjustment as a function of headache diagnosis}

Table 3 displays the prevalence of psychological adjustment characteristics assessed by the SDQ as a function of headache status. Compared with controls (no headache), children with EM were more likely to have the following psychological characteristics (RR and $\mathrm{CI}$ are displayed in the table only for the ease of reading): "Often has temper tantrums or hot tempers" (48.3\% vs. 20.4\%), "Often fights with other children or bullies them" (20.9\% vs. $8 \%)$, "Often unhappy, down-hearted or tearful" (23.3\% vs. 12.3\%), "Easily distracted, concentration wanders" (38.2\% vs. 25.3\%), "Nervous or clingy in new situations, easily loses confidence" (39.3\% vs. 22.1\%), "Picked on or bullied by other children" ( $34.4 \%$ vs. $18.9 \%$ ), and "Many fears, easily scared" (36.3\% vs. 20.6\%; Table 3$)$. Children with ETTH presented no significant differences from controls (Table 3 ).

Figure 1 contrasts the SDQ scores of children with EM and ETTH relative to controls ("no headache").
Compared with controls, children with EM were significantly more likely to have abnormal scores in the following domains: emotional symptoms $(73.9 \%$ vs. $21.6 \%, \mathrm{RR}=3.43,95 \% \mathrm{CI}=2.51-4.69)$, conduct problems $(45.7 \%$ vs. $23.4 \%, \mathrm{RR}=1.96,95 \% \mathrm{CI}=1.37$ $2.79)$, total difficulties $(52.2 \%$ vs. $23.4 \%, \mathrm{RR}=2.23$, $95 \% \mathrm{CI}=1.59-3.13)$, and presence of impact $(12 \%$ vs. $4.2 \%, \mathrm{RR}=2.85,95 \% \mathrm{CI}=1.15-7.11)$. Compared with controls, children with ETTH were significantly more likely to have conduct problems $(34.5 \% v s .23 .4 \%$, RR $=1.48,95 \% \mathrm{CI}=1.02-2.16$ ).

\section{Multivariate analyses}

In the multivariate analyses, gender, race, parents' marital status, parents' educational level, and income class were not significantly associated with SDQ scores in children with EM. The total difficulties score in psychological adjustment was significantly influenced by headache frequency $(p<.05)$, analgesic intake $(p<.001)$, and the occurrence of nausea $(p<$ $.01)$ and vomiting $(p<.05)$ in headache attacks (Table 4). The occurrence of impact caused by difficulties in psychological adjustment demonstrated a nonsignificant trend toward an association with poor school 
Table 2. Prevalence of migraine (overall and episodic), tension-type headache (overall and episodic), and "no headache" as a function of demographics

\begin{tabular}{|c|c|c|c|c|c|c|c|c|c|c|c|c|c|c|c|}
\hline \multirow[b]{2}{*}{ Age } & \multicolumn{3}{|c|}{ No headache } & \multicolumn{3}{|c|}{ Migraine overall } & \multicolumn{3}{|c|}{ pisodic migraine } & \multicolumn{3}{|c|}{$\begin{array}{l}\text { Tension-type } \\
\text { headache } \\
\text { overall }\end{array}$} & \multicolumn{3}{|c|}{$\begin{array}{l}\text { Episodic ten- } \\
\text { sion-type headache }\end{array}$} \\
\hline & $n$ & $\%$ & $\begin{array}{l}\text { RR } \\
(95 \% \mathrm{CI})\end{array}$ & $n$ & $\%$ & $\begin{array}{l}\text { RR } \\
(95 \% \mathrm{CI})\end{array}$ & $n$ & $\%$ & $\begin{array}{l}\text { RR } \\
(95 \% \mathrm{CI})\end{array}$ & $n$ & $\%$ & $\begin{array}{l}\text { RR } \\
(95 \% \mathrm{CI})\end{array}$ & $n$ & $\%$ & $\begin{array}{l}\text { RR } \\
(95 \% \mathrm{CI})\end{array}$ \\
\hline $5-8$ years & 753 & 24.2 & reference & 761 & 24.5 & reference & 223 & 7.2 & reference & 1,368 & 44.0 & reference & 404 & 13.0 & reference \\
\hline $9-12$ years & 417 & 16.3 & $\begin{array}{l}.67 \\
(.60-.75)\end{array}$ & 781 & 30.5 & $\begin{array}{l}1.25 \\
(1.15-1.36)\end{array}$ & 287 & 11.2 & $\begin{array}{l}1.56 \\
(1.32-1.85)\end{array}$ & 1,122 & 43.8 & $\begin{array}{l}1.00 \\
(.94- \\
1.06)\end{array}$ & 322 & 12.6 & $\begin{array}{l}.97 \\
(.84-1.11)\end{array}$ \\
\hline \multicolumn{16}{|l|}{ Gender } \\
\hline Female & 525 & 18.8 & $\begin{array}{l}.84 \\
(.76-.93)\end{array}$ & 770 & 27.6 & $\begin{array}{l}1.03 \\
(.94-1.12)\end{array}$ & 268 & 9.6 & $\begin{array}{l}1.14 \\
(.97-1.35)\end{array}$ & 1,261 & 45.1 & $\begin{array}{l}1.06 \\
(1.00- \\
1.12)\end{array}$ & 357 & 12.8 & $\begin{array}{l}1.00 \\
(.87-1.14)\end{array}$ \\
\hline Male & 645 & 22.4 & reference & 772 & 26.8 & reference & 242 & 8.4 & reference & 1,229 & 42.7 & reference & 369 & 12.8 & reference \\
\hline \multicolumn{16}{|l|}{ Race } \\
\hline White & 778 & 20.6 & reference & 1,000 & 26.5 & reference & 338 & 9.0 & reference & 1,725 & 45.8 & reference & 516 & 13.7 & reference \\
\hline Non-white & 338 & 20.2 & $\begin{array}{l}.98 \\
(.87-1.10)\end{array}$ & 488 & 29.2 & $\begin{array}{l}1.10 \\
(1.004-1.21)\end{array}$ & 158 & 9.4 & $\begin{array}{l}1.05 \\
(.88-1.26)\end{array}$ & 661 & 39.5 & $\begin{array}{l}.86 \\
(.81-.92)\end{array}$ & 184 & 11.0 & $\begin{array}{l}.80 \\
(.69-.94)\end{array}$ \\
\hline $\begin{array}{l}\text { Non- } \\
\text { respondents }\end{array}$ & 54 & 23.5 & $\begin{array}{l}1.14 \\
(.89-1.45)\end{array}$ & 54 & 23.5 & $\begin{array}{l}.88 \\
(.70-1.12)\end{array}$ & 14 & 6.1 & $\begin{array}{l}.68 \\
(.40-1.14)\end{array}$ & 104 & 45.2 & $\begin{array}{l}.99 \\
(.85- \\
1.14)\end{array}$ & 26 & 11.3 & $\begin{array}{l}.83 \\
(.57-1.20)\end{array}$ \\
\hline \multicolumn{16}{|l|}{$\begin{array}{l}\text { Income } \\
\text { class }\end{array}$} \\
\hline$A, B$ & 442 & 21.4 & reference & 505 & 24.4 & reference & 162 & 7.8 & reference & 961 & 46.4 & reference & 297 & 14.4 & reference \\
\hline $\mathrm{C}$ & 561 & 19.6 & $\begin{array}{l}.92 \\
(.82-1.03)\end{array}$ & 811 & 28.4 & $\begin{array}{l}1.16 \\
(1.06-1.28)\end{array}$ & 274 & 9.6 & $\begin{array}{l}1.23 \\
(1.02-1.48)\end{array}$ & 1,264 & 44.3 & $\begin{array}{l}.95 \\
(.90- \\
1.01)\end{array}$ & 365 & 12.8 & $\begin{array}{l}.89 \\
(.77-1.03)\end{array}$ \\
\hline $\mathrm{D}, \mathrm{E}$ & 167 & 22.4 & $\begin{array}{l}1.05 \\
(.90-1.23)\end{array}$ & 226 & 30.3 & $\begin{array}{l}1.24 \\
(1.09-1.42)\end{array}$ & 74 & 9.9 & $\begin{array}{l}1.27 \\
(.97-1.65)\end{array}$ & 265 & 35.5 & $\begin{array}{l}.76 \\
(.69-.85)\end{array}$ & 64 & 8.6 & $\begin{array}{l}.60 \\
(.46-.77)\end{array}$ \\
\hline Total & 1,170 & 20.6 & & 1,542 & 27.2 & & 510 & 9.0 & & 2,490 & 43.9 & & 726 & 12.8 & \\
\hline
\end{tabular}

Table 3. Prevalence of SDQ abnormal scores in children with migraine (overall and episodic), tension-type headache (overall and episodic), and controls ("no headache")

\begin{tabular}{|c|c|c|c|c|c|c|c|c|c|c|c|c|c|c|c|c|c|c|}
\hline \multirow{2}{*}{$\begin{array}{l}\text { SDQ } \\
\text { Score }\end{array}$} & \multicolumn{3}{|c|}{ No headache } & \multicolumn{3}{|c|}{ Migraine overall } & \multicolumn{3}{|c|}{ Episodic migraine } & \multicolumn{3}{|c|}{$\begin{array}{l}\text { Tension-type head- } \\
\text { ache overall }\end{array}$} & \multicolumn{3}{|c|}{$\begin{array}{l}\text { Episodic } \\
\text { tension-type } \\
\text { headache }\end{array}$} & \multicolumn{3}{|c|}{ Overall sample } \\
\hline & $n$ & $\%$ & & $n$ & $\%$ & $\begin{array}{l}\mathrm{RR} \\
(\mathbf{9 5 \%} \\
\mathrm{CI}) \\
\end{array}$ & $n$ & $\%$ & $\begin{array}{l}\text { RR } \\
(95 \% \\
\text { CI }) \\
\end{array}$ & $n$ & $\%$ & $\begin{array}{l}\text { RR } \\
(95 \% \\
\text { CI }) \\
\end{array}$ & $n$ & $\%$ & $\begin{array}{l}\mathrm{RR} \\
(\mathbf{9 5} \% \\
\mathrm{CI}) \\
\end{array}$ & $n$ & $\%$ & $\begin{array}{l}\text { RR } \\
(95 \% \\
\text { CI }) \\
\end{array}$ \\
\hline $\begin{array}{l}\text { Emotional } \\
\text { symptoms }\end{array}$ & 241 & 20.6 & $\begin{array}{l}\text { refer- } \\
\text { ence }\end{array}$ & 860 & 55.8 & $\begin{array}{l}2.71 \\
(2.40- \\
3.06)\end{array}$ & 318 & 62.4 & $\begin{array}{l}3.03 \\
(2.66- \\
3.45)\end{array}$ & 822 & 33.0 & $\begin{array}{l}1.60 \\
(1.41- \\
1.82)\end{array}$ & 200 & 27.5 & $\begin{array}{l}1.34 \\
(1.14- \\
1.57)\end{array}$ & 2,026 & 35.7 & $\begin{array}{l}1.73 \\
(1.54 \\
1.95)\end{array}$ \\
\hline $\begin{array}{l}\text { Conduct } \\
\text { problems }\end{array}$ & 271 & 23.2 & $\begin{array}{l}\text { refer- } \\
\text { ence }\end{array}$ & 621 & 40.3 & $\begin{array}{l}1.74 \\
(1.54- \\
1.96)\end{array}$ & 222 & 43.5 & $\begin{array}{l}1.88 \\
(1.63- \\
2.17)\end{array}$ & 716 & 28.8 & $\begin{array}{l}1.24 \\
(1.10- \\
1.40)\end{array}$ & 184 & 25.3 & $\begin{array}{l}1.09 \\
(.93- \\
1.29)\end{array}$ & 1,726 & 30.4 & $\begin{array}{l}1.31 \\
(1.18 \\
1.47)\end{array}$ \\
\hline $\begin{array}{l}\text { Hyperac- } \\
\text { tivity }\end{array}$ & 164 & 14.0 & $\begin{array}{l}\text { refer- } \\
\text { ence }\end{array}$ & 414 & 26.8 & $\begin{array}{l}1.92 \\
(1.63- \\
2.26)\end{array}$ & 149 & 29.2 & $\begin{array}{l}2.08 \\
(1.71- \\
2.54)\end{array}$ & 439 & 17.6 & $\begin{array}{l}1.26 \\
(1.07- \\
1.48)\end{array}$ & 107 & 14.7 & $\begin{array}{l}1.05 \\
(.84- \\
1.32)\end{array}$ & 1,062 & 18.7 & $\begin{array}{l}1.34 \\
(1.15 \\
1.56)\end{array}$ \\
\hline $\begin{array}{l}\text { Peer } \\
\text { problems }\end{array}$ & 277 & 23.7 & $\begin{array}{l}\text { refer- } \\
\text { ence }\end{array}$ & 485 & 31.5 & $\begin{array}{l}1.33 \\
(1.17- \\
1.51)\end{array}$ & 167 & 32.7 & $\begin{array}{l}1.38 \\
(1.18- \\
1.63)\end{array}$ & 594 & 23.9 & $\begin{array}{l}1.01 \\
(.89- \\
1.14)\end{array}$ & 144 & 19.8 & $\begin{array}{l}.84 \\
(.70- \\
1.00)\end{array}$ & 1,454 & 25.6 & $\begin{array}{l}1.08 \\
(.97- \\
1.21)\end{array}$ \\
\hline $\begin{array}{l}\text { Total } \\
\text { difficulties }\end{array}$ & 244 & 20.9 & $\begin{array}{l}\text { refer- } \\
\text { ence }\end{array}$ & 650 & 42.2 & $\begin{array}{l}2.02 \\
(1.78- \\
2.29)\end{array}$ & 244 & 47.8 & $\begin{array}{l}2.29 \\
(1.99- \\
2.65)\end{array}$ & 656 & 26.3 & $\begin{array}{l}1.26 \\
(1.11- \\
1.44)\end{array}$ & 164 & 22.6 & $\begin{array}{l}1.08 \\
(.91- \\
1.29)\end{array}$ & 1,637 & 28.9 & $\begin{array}{l}1.38 \\
(1.23 \\
1.56)\end{array}$ \\
\hline $\begin{array}{l}\text { Total plus } \\
\text { impact }\end{array}$ & 40 & 3.4 & $\begin{array}{l}\text { refer- } \\
\text { ence }\end{array}$ & 227 & 14.7 & $\begin{array}{l}4.31 \\
(3.10- \\
5.97)\end{array}$ & 86 & 16.9 & $\begin{array}{l}4.93 \\
(3.44- \\
7.07)\end{array}$ & 187 & 7.5 & $\begin{array}{l}2.20 \\
(1.57- \\
3.07)\end{array}$ & 40 & 5.5 & $\begin{array}{l}1.61 \\
(1.05- \\
2.47)\end{array}$ & 471 & 8.3 & $\begin{array}{l}2.43 \\
(1.77 \\
3.33)\end{array}$ \\
\hline Total & 1,170 & & & 1,542 & & & 510 & & & 2,490 & & & 726 & & & 5,671 & & \\
\hline
\end{tabular}


Table 4. Multivariate analyses of determinants of SDQ abnormal scores in children with episodic migraine

\begin{tabular}{|c|c|c|c|c|c|c|c|c|}
\hline \multirow{2}{*}{ SDQ scale } & \multirow{2}{*}{ B } & \multirow{2}{*}{ SE } & \multirow{2}{*}{ Wald } & \multirow{2}{*}{$d f$} & \multirow{2}{*}{ Sig. } & \multirow{2}{*}{$\operatorname{Exp}$} & \multicolumn{2}{|l|}{$95 \% \mathrm{CI}$} \\
\hline & & & & & & & \multirow[t]{2}{*}{ Lower } & \multirow[t]{2}{*}{ Upper } \\
\hline \multicolumn{7}{|l|}{ Emotional symptoms } & & \\
\hline Analgesic intake & & & 27.413 & 2 & .000 & & & \\
\hline $0-1$ vs. 2-9/month & 1.582 & .305 & 26.874 & 1 & .000 & 4.866 & 2.675 & 8.851 \\
\hline $0-1$ vs. $>9 /$ month & 1.463 & .732 & 3.997 & 1 & .046 & 4.318 & 1.029 & 18.114 \\
\hline Nausea & 1.982 & .397 & 24.894 & 1 & .000 & 7.255 & 3.331 & 15.802 \\
\hline \multicolumn{9}{|l|}{ Conduct problems } \\
\hline Analgesic intake & & & 9.835 & 2 & .007 & & & \\
\hline $0-1$ vs. $2-9 /$ month & 1.119 & .370 & 9.148 & 1 & .002 & 3.062 & 1.483 & 6.324 \\
\hline Nausea & 1.856 & .497 & 13.940 & 1 & .000 & 6.395 & 2.414 & 16.940 \\
\hline \multicolumn{9}{|l|}{ Hyperactivity } \\
\hline School achievement & -1.100 & .450 & 5.982 & 1 & .014 & .333 & .138 & .804 \\
\hline Analgesic intake & & & 5.569 & 2 & .062 & & & \\
\hline $0-1$ vs. 2-9/month & 1.171 & .496 & 5.569 & 1 & .018 & 3.226 & 1.220 & 8.532 \\
\hline Nausea & 2.080 & .763 & 7.428 & 1 & .006 & 8.001 & 1.793 & 35.701 \\
\hline \multicolumn{9}{|l|}{ Peer problems } \\
\hline Age & .793 & .385 & 4.233 & 1 & .040 & 2.210 & 1.038 & 4.703 \\
\hline Frequency of attacks & & & 9.249 & 2 & .010 & & & \\
\hline$<4$ vs. $5-9 /$ month & 1.654 & .561 & 8.692 & 1 & .003 & 5.230 & 1.741 & 15.710 \\
\hline Analgesic intake & & & 8.453 & 2 & .015 & & & \\
\hline $0-1$ vs. $2-9 /$ month & 1.126 & .476 & 5.584 & 1 & .018 & 3.082 & 1.212 & 7.840 \\
\hline $0-1$ vs. $>9 /$ month & 2.653 & 1.140 & 5.420 & 1 & .020 & 14.196 & 1.521 & 132.474 \\
\hline Nausea & 1.296 & .616 & 4.426 & 1 & .035 & 3.653 & 1.093 & 12.216 \\
\hline Vomiting & 1.120 & .479 & 5.453 & 1 & .020 & 3.064 & 1.197 & 7.842 \\
\hline \multicolumn{9}{|l|}{ Total difficulties } \\
\hline Frequency of attacks & & & 4.850 & 2 & .088 & & & \\
\hline$>4$ vs. $5-9 /$ month & 1.099 & .517 & 4.522 & 1 & .033 & 3.002 & 1.090 & 8.268 \\
\hline Analgesic intake & & & 14.156 & 2 & .001 & & & \\
\hline $0-1$ vs. $2-9 /$ month & 1.367 & .378 & 13.097 & 1 & .000 & 3.923 & 1.871 & 8.223 \\
\hline $0-1$ vs. $>9 /$ month & 2.039 & 1.004 & 4.122 & 1 & .042 & 7.685 & 1.073 & 55.033 \\
\hline Nausea & 1.561 & .497 & 9.854 & 1 & .002 & 4.764 & 1.797 & 12.625 \\
\hline Vomiting & .799 & .377 & 4.490 & 1 & .034 & 2.223 & 1.062 & 4.654 \\
\hline \multicolumn{9}{|l|}{ Causing impact } \\
\hline School achievement & -1.190 & .612 & 3.782 & 1 & .052 & .304 & .092 & 1.009 \\
\hline Frequency (< 4 vs. 5-9/month) & 1.588 & .718 & 4.892 & 1 & .027 & 4.894 & 1.198 & 19.991 \\
\hline Nausea & 2.325 & 1.059 & 4.815 & 1 & .028 & 10.224 & 1.282 & 81.547 \\
\hline
\end{tabular}

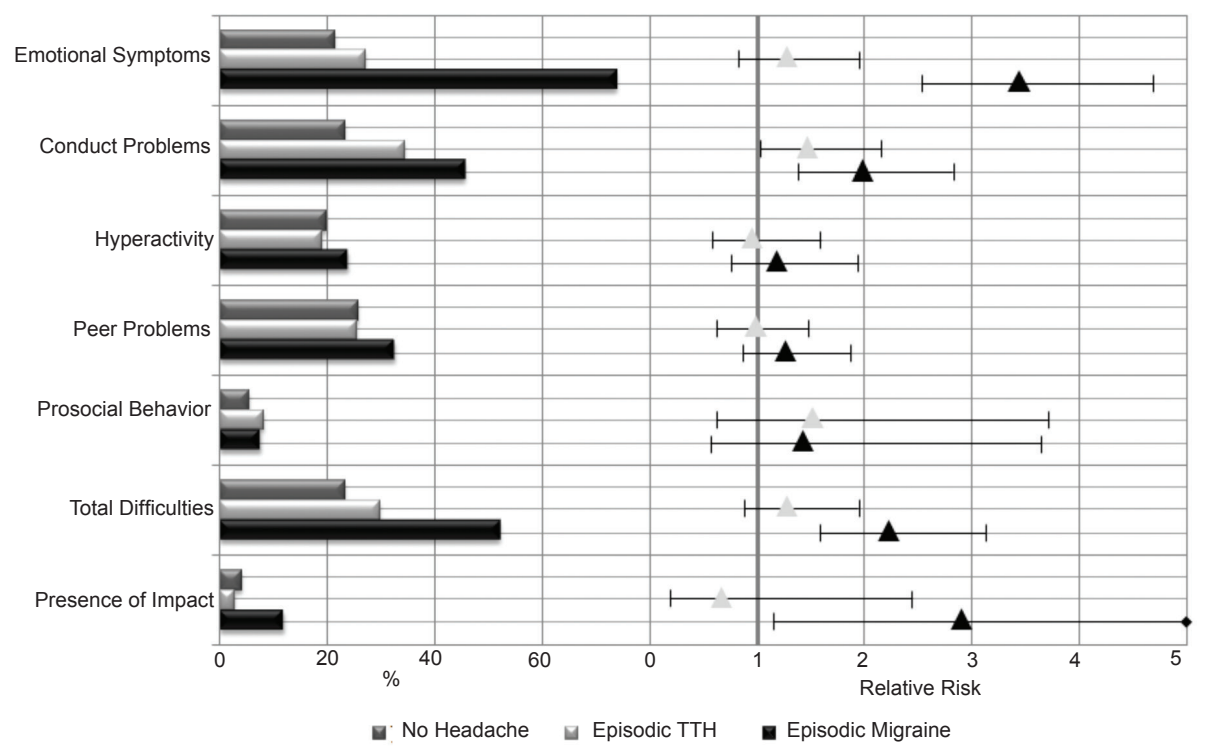

Figure 1. Prevalence of SDQ abnormal scores and relative risk as a function of headache diagnosis. 
achievement $(p=.052)$ and was significantly associated with headache frequency $(p<.05)$ and the occurrence of nausea $(p<.05)$ in headache attacks. Other demographic and migrainous characteristics did not significantly contribute to the model (Table 4).

\section{Discussion}

Migraine in childhood impacts quality of life (Powers, Patton, Hommel, \& Hershey, 2004), school attendance (Abu-Arefeh \& Russell, 1994; Arruda \& Bigal, 2012a), and school achievement (Arruda \& Bigal, 2012a) and is associated with behavioral, emotional, and social problems and competencies (Anttila et al., 2004; Arruda \& Bigal, 2012b; Bruijn et al., 2010). To the best of our knowledge the present study is the first to examine psychological adjustment in preadolescent children with EM and ETTH using the ICHD-2 criteria and SDQ in a large community-based study.

Our findings can be summarized as the following. (1) Relative to controls (children without headache), children with EM were more likely to exhibit the following psychological characteristics that reflect difficulties in psychological adjustment: "Often has temper tantrums or hot tempers," "Often fights with other children or bullies them," "Often unhappy, downhearted or tearful," "Easily distracted, concentration wanders," "Nervous or clingy in new situations, easily loses confidence," "Picked on or bullied by other children," and "Many fears, easily scared." (2) Compared with controls, children with EM were more likely to have abnormal scores in the following domains: emotional symptoms, conduct problems, total difficulties in psychological adjustment, and the presence of impact secondary to these difficulties. (3) Compared with controls, children with ETTH were significantly more likely to have conduct problems. (4) In the multivariate analyses, the total difficulties in psychological adjustment in children with EM were significantly influenced by headache frequency, analgesic intake, and the occurrence of nausea and vomiting in headache attacks. The occurrence of impact caused by difficulties in psychological adjustment was significantly influenced by poor school achievement, headache frequency, and nausea in headache attacks. Other migrainous and demographic characteristics did not significantly contribute to the model.

Two main approaches have been used to assess behavioral symptoms in childhood: categorical diagnoses that describe psychopathological states as distinct syndromes and dimensional approaches that view psychopathology as a deviance from normal with no clear threshold between subjects with and without a disorder (Schmeck et al., 2001). The latter approach is used by the SDQ and was adopted by us. An advantage of this approach is that it avoids stigmatization and labeling, which are common risks when conducting behavioral research.

The SDQ is designed to measure psychological adjustment in children and adolescents. Psychometric evaluations of the instrument have shown satisfactory convergent and discriminant validity, whereas factor analyses have shown mixed results across countries (Stone, Otten, Engels, Vermulst, \& Janssens, 2010).

Our findings corroborate the higher prevalence of emotional symptoms in preadolescent children with EM compared with those without headache. Previous populational studies have also shown a higher prevalence of somatic (Anttila, 2004; Laurell, Larsson, \& EegOlofsson, 2005) and anxiety-depressive symptoms (Fichtel \& Larsson, 2002; Virtanen et al., 2004) in children and adolescents with migraine compared with children without chronic headaches, although controversial findings have been reported concerning children with tension-type headaches (Anttila et al., 2004; Laurell, Larsson, \& Eeg-Olofsson, 2004).

In our sample, children with EM were more likely to exhibit social behaviors and conduct problems directly related to bullying which, in turn, are associated with emotional problems and deeply related to an important impact on personal and social life. A recent meta-analysis of clinical studies strongly suggested that children with migraine are not more likely to have problems in thought, social, delinquent, and aggressive domains relative to children without migraine, but they are more likely to have somatic complaints and exhibit internalizing behavior (Bruijn et al., 2010). Our data, therefore, are consistent with previous findings.

The impact of migraine on psychological adjustment in children is rarely documented, and multivariate analyses with adjustments that account for demographics, family income, school achievement, migraine symptoms, and frequency have not been conducted to the best of our knowledge. This is important to disentangle the true drivers of psychological adjustment in children with migraine.

After adjustments, our findings indicate that headache frequency, nausea, vomiting, and analgesic intake were the key determinants of impact. Headache, nausea, and vomiting are considered some of the most frequent functional-somatic symptoms in children and adolescents associated with young-adult psychopathology (Steinhausen, Eschmann, \& Metzke, 2007).

The strengths and limitations of our study deserve comment. The strengths include the following: (1) the population nature of our study, (2) the relatively large sample size of preadolescents, (3) the use of validated questionnaires, (4) the strict accordance with standardized criteria for headache diagnosis, (5) the relatively high participation rate, and (6) the use of multivariate adjustments.

The limitations include the fact that headache diagnosis was determined by the information provided by the mother. No direct interview was conducted with the child. Although this is not different from what is often seen in medical practice for young children, the potential biases of our method need to be explored (Arruda, Bordini, Ciciarelli, \& Speciali, 2004). We may also have underestimated the prevalence of 
ETTHs because we only classified the most severe type of headache presented by patients. This is a standard method in the field (Lipton, Stewart, \& Simon, 1998); however, by not assessing more than one type of headache, we cannot adjust for whether one headache (ETTH) influences the association of headache/SDQ scores in children with another type of headache (EM). Another limitation is that the ICHD-2 may have limited value in separating migraine and tension-type headache in young children. In a longitudinal study with 417 children, the ICHD-2 had high specificity but low sensitivity in diagnosing migraine in childhood (Arruda et al., 2004). Finally, we identified children registered in the public school system. Although this is a well-established method for investigating the epidemiology of diseases in the pediatric population (Abu-Arefeh, \& Russell, 1994; Bille, 1997) because education is mandatory in most countries, children from higher socioeconomic strata are less likely to be enrolled in the public school system. Adjustments are not able to precisely address this issue because all socioeconomic variables would be shifted toward the lower incomes in samples of children from public school only.

In summary, we found that EM was significantly associated with difficulties in psychological adjustment in preadolescent children. Specifically, children with migraine were more likely to present emotional and conduct symptoms that impact their ability to master to some need or stress in the environment. The association between these psychological difficulties and the burden caused by migraine itself may perpetuate both conditions. Providers should be aware of this possibility in children with migraine to properly address the problem. Long-term longitudinal studies are needed to study causality, investigate predictive validity, and clarify the effect of prophylactic treatment in psychological adjustment in children with migraine.

\section{References}

Abu-Arafeh, I., Razak, S., Sivaraman, B., \& Graham, C. (2010). Prevalence of headache and migraine in children and adolescents: A systematic review of population-based studies. Developmental Medicine and Child Neurology, 52(12), 1088-1097.

Abu-Arefeh, I., \& Russell, G. (1994). Prevalence of headache and migraine in schoolchildren. British Medical Journal, 309(6957), 765-769.

Akinbami, L. J., Liu, X., Pastor, P. N., \& Reuben, C. A. (2011). Attention deficit hyperactivity disorder among children aged 5-17 years in the United States, 1998-2009. NCHS Data Brief, 70, 1-8.

American Psychiatric Association (1994). Diagnostic and statistical manual of mental disorders, 4th edition. Washington, D.C.: American Psychiatric Press.

Andrasik, F., Kabela, E., Quinn, S., Attanasio, V., Blanchard, E. B., \& Rosenblum, E. L. (1988). Psychological functioning of children who have recurrent migraine. Pain, 34(1), 43-52.

Anttila, P. (2004). Tension-type headache in children and adolescents. Current Pain and Headache Reports, 8(6), 500-504.

Anttila, P., Sourander, A., Metsahonkala, L., Aromaa, M., Helenius, H., \& Sillanpaa, M. (2004). Psychiatric symptoms in children with primary headache. Journal of the American Academy of Child and Adolescent Psychiatry, 43(4), 412-419.
Arruda, M. A. (2009). Aprender Criança. [www.aprendercrianca.com. br; accessed October 14, 2013]

Arruda, M. A., \& Bigal, M. E. (2012a). Migraine and migraine subtypes in pre-adolescent children: association with school performance. Neurology, 79(18), 1881-1888.

Arruda, M. A., \& Bigal, M. E. (2012b). Behavioral and emotional symptoms and primary headaches in children: a population-based study. Cephalalgia, 32(15), 1093-1100.

Arruda, M. A., Bordini, C. A., Ciciarelli, M. C., \& Speciali, J. G. (2004). Decreasing the minimal duration of the attack to 1 hour: Is this sufficient to increase the sensitivity of the ICHD-II diagnostic criteria for migraine in childhood? Journal of Headache and Pain, 5(2), 131-136.

Arruda, M. A., Guidetti, V., Galli, F., Albuquerque, R. C., \& Bigal, M. E. (2010a). Primary headaches in childhood: A population-based study. Cephalalgia, 30(9), 1056-1064.

Arruda, M. A., Guidetti, V., Galli, F., Albuquerque, R. C., \& Bigal, M. E. (2010b). Migraine, tension-type headache, and attention-deficit/ hyperactivity disorder in childhood: A population-based study. Postgraduate Medicine, 122(5), 18-26.

Bigal, M., Krymchantowski, A. V., \& Lipton, R. B. (2009). Barriers to satisfactory migraine outcomes: What have we learned, where do we stand? Headache, 49(7), 1028-1041.

Bille, B. (1962). Migraine in school children: a study of the incidence and short-term prognosis, and a clinical, psychological and electroencephalographic comparison between children with migraine and matched controls. Acta Paediatrica Suppplementum, $136,1-151$.

Bille, B. (1997). A 40-year follow-up of school children with migraine. Cephalalgia, 17(4), 488-491; discussion 487.

Bruijn, J., Locher, H., Passchier, J., Dijkstra, N., \& Arts, W. F. (2010). Psychopathology in children and adolescents with migraine in clinical studies: A systematic review. Pediatrics, 126(2), 323-332.

Dickey, W. C., \& Blumberg, S. J. (2004). Revisiting the factor structure of the Strengths and Difficulties Questionnaire: United States, 2001. Journal of the American Academy of Child and Adolescent Psychiatry, 43(9), 1159-1167.

Epstein, J. N., Langberg, J. M., Lichtenstein, P. K., Kolb, R., Altaye, M., \& Simon, J. O. (2011). Use of an Internet portal to improve community-based pediatric ADHD care: A cluster randomized trial. Pediatrics, 128(5), E1201-E1208.

Fichtel, A., \& Larsson, B. (2002). Psychosocial impact of headache and comorbidity with other pains among Swedish school adolescents. Headache, 42(8), 766-775.

Fleitlich-Bilyk, B., \& Goodman, R. (2004). Prevalence of child and adolescent psychiatric disorders in southeast Brazil. Journal of the American Academy of Child and Adolescent Psychiatry, 43(6), 727734.

Galli, F., Canzano, L., Scalisi, T. G., \& Guidetti, V. (2009). Psychiatric disorders and headache familial recurrence: A study on 200 children and their parents. Journal of Headache and Pain, 10(3), 187-197.

Galli, F., D’Antuono, G., Tarantino, S., Viviano, F., Borrelli, O., Chirumbolo, A., Cucchiara, S., \& Guidetti, V. (2007). Headache and recurrent abdominal pain: A controlled study by the means of the Child Behaviour Checklist (CBCL). Cephalalgia, 27(3), 211-219.

Goodman, A., Lamping, D. L., \& Ploubidis, G. B. (2010). When to use broader internalizing and externalizing subscales instead of the hypothesized five subscales on the Strengths and Difficulties Questionnaire (SDQ): Data from British parents, teachers and children. Journal of Abnormal Child Psychology, 38(8), 1179-1191.

Goodman, J. E., \& McGrath, P. J. (1991). The epidemiology of pain in children and adolescents: a review. Pain, 46(3), 247-264.

Goodman, R., Neves dos Santos, D., Robatto Nunes, A. P., Pereira de Miranda, D., Fleitlich-Bilyk, B., \& Almeida Filho, N. (2005). The Ilha de Maré study: A survey of child mental health problems in a predominantly African-Brazilian rural community. Social Psychiatry and Psychiatric Epidemiology, 40(1), 11-17.

Guidetti, V., Galli, F., Fabrizi, P., Giannantoni, A. S., Napoli, L., Bruni, O., \& Trillo, S. (1998). Headache and psychiatric comorbidity: clinical aspects and outcome in an 8-year follow-up study. Cephalalgia, 18(7), 455-462.

Headache Classification Subcommittee of the International Headache Society (2004). The International Classification of Headache Disorders: 2nd edition. Cephalalgia, 24(Suppl. 1), 9-160. 
Instituto Brasileiro de Geografia e Estatística (2009). Estatística da População Brasileira. [http://www.ibge.gov.br/home/estatistica/ populacao/estimativa2008/POP2008_DOU.pdf; accessed October $14,2013]$.

Lanzi, G., Zambrino, C. A., Ferrari-Ginevra, O., Termine, C., D’Arrigo, S., Vercelli, P., De Silvestri, A., \& Guglielmino, C. R. (2001). Personality traits in childhood and adolescent headache. Cephalalgia, 21(1), 53-60.

Laurell, K., Larsson, B., \& Eeg-Olofsson, O. (2004). Prevalence of headache in Swedish schoolchildren, with a focus on tension-type headache. Cephalalgia, 24(5), 380-388.

Laurell, K., Larsson, B., \& Eeg-Olofsson, O. (2005). Headache in schoolchildren: Association with other pain, family history and psychosocial factors. Pain, 119(1-3), 150-158.

Lavigne, J. V., \& Faier-Routman, J. (1992). Psychological adjustment to pediatric physical disorders: A meta-analytic review. Journal of Pediatric Psychology, 17(2), 133-157.

Lipton, R. B., Diamond, S., Reed, M., Diamond, M. L., \& Stewart, W. F. (2001). Migraine diagnosis and treatment: results from the American Migraine Study II. Headache, 41(7), 638-645.

Lipton, R. B., \& Silberstein, S. D. (1994). Why study the comorbidity of migraine? Neurology, 44(10 Suppl. 7), S4-S5.

Lipton, R. B., Stewart, W. F., \& Simon, D. (1998). Medical consultation for migraine: Results from the American Migraine Study. Headache, 38(2), 87-96.

Mazzone, L., Vitiello, B., Incorpora, G., \& Mazzone, D. (2006). Behavioural and temperamental characteristics of children and adolescents suffering from primary headache. Cephalalgia, 26(2), 194-201.

Perquin, C. W., Hunfeld, J. A., Hazebroek-Kampschreur, A. A., van Suijlekom-Smit, L. W., Passchier, J., Koes, B. W., \& van der Wouden, J. C. (2001). Insights in the use of health care services in chronic benign pain in childhood and adolescence. Pain, 94(2), 205-213.

Powers, S. W., Patton, S. R., Hommel, K. A., \& Hershey, A. D. (2003). Quality of life in childhood migraines: Clinical impact and comparison to other chronic illnesses. Pediatrics, $112(1 \mathrm{Pt}$ 1), e1-e5.
Powers, S. W., Patton, S. R., Hommel, K. A., \& Hershey, A. D. (2004). Quality of life in paediatric migraine: Characterization of age-related effects using PedsQL 4.0. Cephalalgia, 24(2), 120127.

Queiroz, L. P., Peres, M. F., Kowacs, F., Piovesan, E. J., Ciciarelli, M. C., Souza, J. A., \& Zukerman, E. (2008). Chronic daily headache in Brazil: A nationwide population-based study. Cephalalgia, 28(12), 1264-1269.

Saper, J. R. (1983). Headache disorders: Current concepts and treatment strategies. Boston: John Wright.

Sarbin, T. R. (1940). Adjustment on psychology. Character and Personality, 8(3), 240-249.

Schmeck, K., Poustka, F., Dopfner, M., Pluck, J., Berner, W., Lehmkuhl, G., Fegert, J. M., Lenz, K., Huss, M., \& Lehmkuhl, U. (2001). Discriminant validity of the child behaviour checklist CBCL-4/18 in German samples. European Child and Adolescent Psychiatry, 10(4), 240-247.

Steinhausen, H. C., Eschmann, S., \& Metzke, C. W. (2007). Continuity, psychosocial correlates, and outcome of problematic substance use from adolescence to young adulthood in a community sample. Child and Adolescent Psychiatry and Mental Health, 1(1), 12.

Stone, L. L., Otten, R., Engels, R. C., Vermulst, A. A., \& Janssens, J. M. (2010). Psychometric properties of the parent and teacher versions of the strengths and difficulties questionnaire for 4- to 12-year-olds: a review. Clinical Child and Family Psychology Review, 13(3), 254-274.

Vannatta, K., Getzoff, E. A., Powers, S. W., Noll, R. B., Gerhardt, C. A., \& Hershey, A. D. (2008). Multiple perspectives on the psychological functioning of children with and without migraine. Headache, 48(7), 994-1004.

Virtanen, R., Aromaa, M., Koskenvuo, M., Sillanpaa, M., Pulkkinen, L., Metsahonkala, L., ... Kaprio, J. (2004). Externalizing problem behaviors and headache: a follow-up study of adolescent Finnish twins. Pediatrics, 114(4), 981-987. 